\title{
ESTUDO DO PROCESSO DE SEPARAÇÃO POR MEMBRANAS POLÍMERICAS EM SOLUÇÕES SINTÉTICAS DE CORANTE EMPREGADAS NO TINGIMENTO DE PELE DE PEIXE
}

\author{
F. A. LEITE ${ }^{1}$, S. D. P. MASSOCHIN ${ }^{1}$, L. D. FIORENTIN², A. N. MÓDENES ${ }^{2}$ \\ ${ }^{1}$ Universidade Estadual do Oeste do Paraná, Faculdade de Engenharia Química \\ ${ }^{2}$ Universidade Estadual do Oeste do Paraná, Departamento de Engenharia Química \\ E-mail para contato: fabioaugustoleite1995@gmail.com
}

\begin{abstract}
RESUMO - A transformação da pele peixe em couro, gera elevadas quantidades de resíduos líquidos, que quando descartados sem o devido tratamento, causam sérios danos ao ecossistema. Desta forma, este trabalho teve como objetivo realizar o tratamento de duas soluções sintéticas de corante amarelo reativo e preto direto, empregados no tingimento da pele de peixe, utilizando o método de separação por membranas poliméricas. As membranas foram compostas dos polímeros polietersulfona (PES) e polisulfona (PSU) e sais $\left(\mathrm{NaCl}, \mathrm{KCl}\right.$ e $\left.\mathrm{CaCl}_{2}\right)$, dissolvidos em N'N Dimetilformamida e confeccionadas pelo método de inversão de fases. Determinou-se a permeabilidade hidráulica com água (Mili-Q) e com soluções sintéticas de corante (50 ppm), bem como, o tamanho médio dos poros da membrana, a porcentagem de remoção do corante e o índice de fouling. Os resultados mostraram que todas as membranas apresentaram tamanho de poro médio igual a $50 \AA$, característicos de membranas de ultrafiltração. A membrana composta de $16,4 \%$ PSU, $5 \%$ PES e 3\% $\mathrm{KCl}$ apresentou os maiores resultados em relação aos parâmetros avaliados para o corante amarelo reativo. Enquanto que a membrana a $16,4 \%$ PSU e $3 \% \mathrm{NaCl}$ foi a que se sobressaiu para a solução do corante preto direto. Desta forma, a metodologia pode ser utilizada no tratamento dos corantes empregados no tingimento da pele de peixe.
\end{abstract}

\section{INTRODUÇÃO}

O crescimento urbano tem acarretado um aumento na produção alimentícia no Brasil. Desta forma, uma enorme quantidade de água é utilizada, seja para produção de alimentos ou outros produtos quaisquer, com geração de elevadas quantidades de efluentes que podem contaminar o meio ambiente. Em muitos destes efluentes, há a presença de corantes facilmente identificados a olho nu (CHAGURI, 2010). Entre estes produtos está o curtimento e tingimento da pele de peixe.

O Brasil exige das indústrias, conforme norma CONAMA, de 1996 (n²0, de 18 de junho de 1996), que estes efluentes tenham tratamento prévio antes de serem descartados em vias pluviais, pois causam a diminuição da capacidade de aeração e impedem a penetração da luz solar nos rios e lagos (STREIT, 2011). Para o tratamento destes tipos de efluentes, diversas são as alternativas, tais como: precipitação química e processos de óxido-redução, lodo ativado, filtração e adsorção, o qual é o mais empregado na atualidade. Entretanto, tecnologias 
limpas têm se destacado, como é o caso dos processos de separação por membranas (PALOMINO-ROMERO, et. al. 2011).

As membranas possuem a capacidade de controlar as taxas de transporte relativo a várias espécies presentes em um resíduo líquido, produzindo um permeado pobre em concentração de uma determinada espécie e um retentado com grandes concentrações da mesma espécie ou de outras (OURADI et al, 2014, HABERT et al., 2006). As membranas são classificadas segundo a sua composição e o método de atuação, que originam as membranas poliméricas e inorgânicas (BEZERRA, et. al.2014).

Desta forma, este trabalho teve como objetivo a confecção de membranas poliméricas de diferentes composições a partir da técnica de inversão de fases e aplicadas no tratamento de uma solução sintética de diferentes corantes empregada no tingimento de peles de peixe.

\section{MATERIAIS E MÉTODOS}

\subsection{Composições das membranas}

Com base em estudos realizados na literatura, optou-se por avaliar o comportamento dos polímeros polisulfona (PSU) e polietersulfona (PES), dissolvidos em N`N Dimetilformamida, juntamente com os cloreto de sódio, cálcio e potássio em diferentes composições, Tabela 1.

Tabela 1 - Composições das membranas poliméricas

\begin{tabular}{cccccc}
\hline Composição & PES (\%) & PSU $(\%)$ & $\mathrm{KCl}(\%)$ & $\mathrm{NaCl}(\%)$ & $\mathrm{CaCl}_{2}(\%)$ \\
\hline M1 & 5 & 16,4 & 3 & - & - \\
M2 & - & 16,4 & 3 & - & - \\
M3 & 5 & 16,4 & - & 3 & - \\
M4 & - & 16,4 & - & 3 & - \\
M5 & 5 & 16,4 & - & - & 3 \\
\hline
\end{tabular}

\subsection{Confecção das Membranas Poliméricas e Módulo de Filtração}

Preparou-se $30 \mathrm{~mL}$ de solução polimérica em um erlenmeyer na concentração desejada. Em seguida vedou-se o recipiente, submetendo-o a agitação, de 8 a $25 \mathrm{~h}$, a $34 \pm 2{ }^{\circ} \mathrm{C}$. A solução permaneceu em repouso por $48 \mathrm{~h}$. O espalhamento da solução foi realizado com auxílio de um bastão de vidro sobre uma placa de vidro que continha uma folha de poliéster com fios de nylon controlando a espessura. Em seguida a placa foi submersa em um recipiente com água Mili-Q por $4 \mathrm{~h}$, sendo retirada em seguida para secagem por $24 \mathrm{~h}$. O módulo de filtração é composto de uma célula em forma de cilindro (aço inoxidável), com volume aproximado de $300 \mathrm{~mL}$, e força motriz dada por um cilindro de nitrogênio acoplado ao módulo, com válvula reguladora de pressão. A membrana é inserida na base do módulo e possui área útil filtrante de $2,5.10^{-4} \mathrm{~m}^{2}$. A alimentação é realizada de forma batelada e o permeado é coletado em intervalos de tempos pré-determinados.

\subsection{Processo de Caracterização da Membrana}

Compactação e pressão de operação: A compactação das membranas, se deu pela passagem de água Mili-Q durante $2 \mathrm{~h}$ a 1 bar. Em seguida, determinou-se a pressão de 
operação $\left(\mathrm{P}_{\mathrm{op}}\right)$ com água Mili-Q, em que se aumentou gradativamente a pressão (limite da célula 4 bar), até que o fluxo não variasse mais e com os dados dessa etapa determinou-se o fluxo limpo. O fluxo permeado foi calculado pela Equação 1, sendo a $\mathrm{P}_{\mathrm{op}}$ determinada como sendo o maior valor de fluxo estabilizado obtido.

$$
\mathrm{J}=\frac{\mathrm{m}_{\mathrm{p}}}{\Delta \mathrm{t} \mathrm{A}}
$$

Em que: $\mathrm{J}$ é o fluxo permeado $\left(\mathrm{kg} \mathrm{h}^{-1} \mathrm{~m}^{-2}\right), \mathrm{m}_{\mathrm{p}}$ a massa de permeado coletada no intervalo de tempo $(\mathrm{kg}), \Delta \mathrm{t}$ a variação de tempo (h) e $\mathrm{A}$, a área útil da membrana $\left(\mathrm{m}^{2}\right)$.

Passagem da solução de albumina bovina - BSA: Preparou-se a solução de albumina bovina (40 $\AA$ ), a 600 ppm e passou-se pela membrana na $\mathrm{P}_{\text {op }}$. Por meio da curva de calibração $(210 \mathrm{~nm})$, determinou-se a concentração do permeado e com uso das Equações 2 (LOWRY, et. al. 1951) e 3 (BASSETI, 2002) determinou-se o raio aparente de cada membrana.

$$
\begin{aligned}
& C_{\left.R^{(} \%\right)}=\left(1-\frac{C_{p, B S A}}{C_{C, B S A}}\right) 100 \\
& C_{R}(\%)=\left(\frac{r_{S}}{r}\right) 100
\end{aligned}
$$

Em que: $C_{R}$ é o coeficiente de retenção, $C_{C, B S A}$ é a concentração de moléculas presentes no concentrado/retentado (ppm), $\mathrm{C}_{\mathrm{p}, \mathrm{BSA}}$ é a concentração de permeado (ppm), $\mathrm{r}_{\mathrm{S}}$ é o raio médio do soluto $(\AA)$ e r é o raio médio dos poros da membrana $(\AA)$.

Soluções sintéticas de corante determinação do índice de fouling: Preparou-se uma solução sintética $(50 \mathrm{ppm})$ empregando os corantes amarelo reativo e preto direto dissolvidos em água Mili-Q. Realizou-se a varredura do comprimento de onda e construiu-se a curva de calibração de cada um deles. Em seguida, passou-se a solução de corante em cada membrana na $\mathrm{P}_{\mathrm{op}}$, até fluxo constante. A concentração do permeado foi determinada, pela curva de calibração pré-determinada no comprimento de onda, 410 e $480 \mathrm{~nm}$, para as soluções sintéticas do amarelo reativo e preto direto, respectivamente. Em seguida, passou-se água Mili-Q na mesma $\mathrm{P}_{\mathrm{op}}$ até fluxo constante, determinando-se assim o fouling, Equação 4.

$$
F \%)=\left(\frac{J_{\text {lim po }}-J_{\text {final }-\mathrm{H} 2 \mathrm{O}}}{J_{\text {lim po }}}\right) 100
$$

Em que: $\mathrm{F}(\%)$ é percentual de índice de fouling (\%), $\mathrm{J}_{\text {limpo }}$ o fluxo permeado obtido com água Mili-Q na $\mathrm{P}_{\text {op }}\left(\mathrm{kg} \mathrm{h}^{-1} \mathrm{~m}^{-2}\right)$ e $\mathrm{J}_{\text {final-H2O }}$ o fluxo permeado obtido com água Mili-Q, após a passagem da solução de corante na $\mathrm{P}_{\mathrm{op}}\left(\mathrm{kg} \mathrm{h}^{-1} \mathrm{~m}^{-2}\right)$.

\section{RESULTADOS E DISCUSSÃO}

\subsection{Determinação da Pressão de Operação}


Após a compactação de cada uma das membranas, procurou-se determinar a $\mathrm{P}_{\mathrm{op}}$ com água Mili-Q. A Tabela 2 apresenta os fluxos permeados estabilizados em cada uma das pressões.

Tabela 2 - Pressão de operação com água Mili-Q

\begin{tabular}{ccccc}
\hline Composição & $\mathrm{P}=1,0$ bar & $\mathrm{P}=2,0$ bar & $\mathrm{P}=3,0$ bar & $\mathrm{P}=4,0$ bar \\
\cline { 2 - 5 } & $\mathrm{J}\left(\mathrm{kg} \mathrm{h}^{-1} \mathrm{~m}^{-2}\right)$ & ${\mathrm{J}\left(\mathrm{kg} \mathrm{h}^{-1} \mathrm{~m}^{-2}\right)}_{\mathrm{J}\left(\mathrm{kg} \mathrm{h}^{-1} \mathrm{~m}^{-2}\right)}$ & $\mathrm{J}\left(\mathrm{kg} \mathrm{h}^{-1} \mathrm{~m}^{-2}\right)$ \\
\hline M1 & 22,9 & 70,2 & 46,0 & --- \\
M2 & 20,6 & 30,1 & 9,6 & --- \\
M3 & 14,9 & 26,9 & 22,2 & --- \\
M4 & 15,3 & 11,3 & --- & --- \\
M5 & 43,3 & 77,1 & 107,0 & 117,4 \\
\hline
\end{tabular}

Observa-se na Tabela 2, que se tem um aumento gradativo do fluxo estabilizado em função da pressão, até se atingir o limite de pemeação de cada membrana. Desta forma, notase que para as membranas M1, M2 e M3 a $\mathrm{P}_{\text {op }}$ é 2 bar, enquanto que para M4 e M5, é 1 e 4 bar, respectivamente. Pode-se ainda perceber, que a membrana M5, possui o maior fluxo permeado e maior $\mathrm{P}_{\mathrm{op}}$ entre as composições estudadas, possivelmente devido a influência do $\mathrm{CaCl}_{2}$. Já para a membrana confeccionada com $\mathrm{NaCl}$ (M4), se obteve os piores valores de fluxo e menor $\mathrm{P}_{\mathrm{op}}$. Vale ressaltar também, que o comportamento das curvas de fluxo permeado em função do tempo foram característicos dos processo de separação pro membrana, ou seja, iniciando com valores elevados e diminuindo com o tempo, até estabilização.

\subsection{Determinação dos raios aparentes}

A Tabela 3, apresenta o fluxo estabilizado para a albumina obtido na $\mathrm{P}_{\text {op }}$ de cada membrana, bem como a concentração final do permeado, com os valores dos raios aparentes, calculados por meio das Equações 2 e 3.

Tabela 3 - Raio médio dos poros das membranas

\begin{tabular}{cccccc}
\hline Composição & $\mathrm{P}_{\mathrm{op}}(\mathrm{bar})$ & $\mathrm{J}\left(\mathrm{kg} \cdot \mathrm{h}^{-1} \mathrm{~m}^{-2}\right)$ & $\mathrm{C}_{\mathrm{p}, \mathrm{BSA}}\left(\mathrm{mgL}^{-1}\right)$ & $\mathrm{C}_{\mathrm{R}}(\%)$ & $\mathrm{r}(\AA)$ \\
\hline $\mathrm{M} 1$ & 2 & 20,8 & 126,1 & 79,0 & 50,6 \\
M2 & 2 & 8,3 & 130,2 & 78,3 & 51,1 \\
M3 & 2 & 17,7 & 123,3 & 79,4 & 50,3 \\
M4 & 1 & 2,3 & 129,2 & 78,5 & 51,0 \\
M5 & 4 & 20,6 & 126,8 & 78,9 & 50,7 \\
\hline
\end{tabular}

Nota-se pela Tabela 3, que as membranas M1 e M5, obtiveram os maiores valores de fluxo permeado. Em termos de concentração final, a membrana M2, foi a que apresentou um leve aumento da remoção de cor em relação às demais. Entretanto, pode-se visualizar que apesar das diferentes concentrações empregando os três sais, a $\mathrm{C}_{\mathrm{p}, \mathrm{BSA}}$ manteve-se praticamente constante para todas as membranas. Com a obtenção dos valores dos raios médios de cada membrana e com o auxílio de HABERT et. al., 2006, pode-se concluir que todas as membranas são classificadas como sendo de ultrafiltração, ou seja, membranas que 
empregam gradientes de pressão entre 0,5-7 bar, com raios médios de poros 10 - $1000 \AA$, sendo indicada principalmente para processos de fracionamento e concentração de proteínas, recuperação de pigmentos e de óleos.

\subsection{Passagem da Solução Sintética de Corante e Taxa de Fouling}

Após submeter às membranas as soluções de corantes na $\mathrm{P}_{\mathrm{op}}$, determinou-se a porcentagem de remoção do corante $(\mathrm{R} \%)$ e o cálculo do índice de fouling em cada membrana (Equação 4). Os resultados são ilustrados na Tabela 4.

Tabela 4 - Fluxos estáveis com soluções sintéticas e estudo do índice de fouling

\begin{tabular}{ccccc|cccc}
\hline & \multicolumn{2}{c}{ Amarelo reativo } & \multicolumn{2}{c|}{ fouling } & \multicolumn{2}{c}{ Preto direto } & \multicolumn{2}{c}{ fouling } \\
\hline Composição & $\begin{array}{c}\mathrm{J} \cdot \\
\left(\mathrm{kg} \cdot \mathrm{h}^{-1} \mathrm{~m}^{-2}\right)\end{array}$ & $\begin{array}{c}\mathrm{R} \\
(\%)\end{array}$ & $\begin{array}{c}\mathrm{J}_{\text {final-H2O }} \\
\left(\mathrm{kg} \cdot \mathrm{h}^{-1} \mathrm{~m}^{-2}\right)\end{array}$ & $\begin{array}{c}F \\
(\%)\end{array}$ & $\begin{array}{c}\mathrm{J} \\
\left(\mathrm{kg} \cdot \mathrm{h}^{-1} \mathrm{~m}^{-2}\right)\end{array}$ & $\begin{array}{c}\mathrm{R} \\
(\%)\end{array}$ & $\begin{array}{c}\mathrm{J}_{\text {final-H2O }} \\
\left(\mathrm{kg} \cdot \mathrm{h}^{-1} \mathrm{~m}^{-2}\right)\end{array}$ & $\begin{array}{c}F \\
(\%)\end{array}$ \\
\hline M1 & 40,2 & 7,1 & 44,5 & 30,7 & 45,4 & 56,3 & 55,7 & 26,8 \\
M2 & 11,3 & 35,7 & 8,1 & 73,4 & 3,1 & 82,6 & 7,5 & 80,3 \\
M3 & 63,7 & 47,1 & 20,8 & 19,6 & 56,4 & 84,8 & 22,9 & 18,3 \\
M4 & 26,0 & 7,1 & 28,1 & 36,6 & 16,9 & 89,5 & 27,9 & 6,3 \\
M5 & 156,8 & 27,9 & 108,0 & 11,1 & 77,9 & 45,9 & 177,6 & 31,3 \\
\hline
\end{tabular}

Nota-se na Tabela 3, que os fluxos permeados com a solução do corante amarelo reativo obtiveram maiores remoções, quando comparados ao preto direto. As maiores remoções foram obtidas pelas membranas M3 e M4 para os corantes sintéticos, amarelo e preto, com remoções aproximadas de 50 e $90 \%$, respectivamente. Em relação ao fluxo permeado com corante, as membranas de composição M5 e M3 apresentaram os maiores valores, sendo eles de aproximadamente 157 e $64 \mathrm{~kg} \mathrm{~h}^{-1} \mathrm{~m}^{-2}$, para o amarelo reativo, respectivamente. Enquanto que para o corante preto, as maiores remoções também foram para as membranas M5 e M3, com fluxos de 78 e $56 \mathrm{~kg} \mathrm{~h}^{-1} \mathrm{~m}^{-2}$, respectivamente.

Em relação ao estudo do índice de fouling, observa-se que os fluxos obtidos com o $\mathrm{J}_{\text {final- }}$

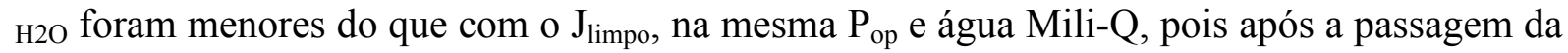
solução sintética dos corantes, se tem o entupimento dos poros das membranas. As composições que apresentaram os menores índices de fouling foram M5 e M3, com F(\%) de 11,1 e $19,6 \%$, para a solução sintética de amarelo reativo, respectivamente. Consequentemente, estas foram às membranas que apresentaram os maiores valores de fluxo permeado com o corante. Por sua vez, para a solução sintética do corante preto direto, as composições M4 e M3 demonstraram os melhores resultados, sendo eles 6,25 e 18,3\% respectivamente.

No entanto, os resultados obtidos para o índice de fouling na membrana M2, mostraram-se mais significativo, com valores em torno de 73 e $80 \%$, para ambas as soluções sintéticas trabalhadas. Pode-se notar ainda, que as membranas M1 e M5, apresentaram valores intermediários (aproximadamente 30\%) somente para a solução sintética do corante preto direto. Para a reutilização das membranas, seria necessária a realização de limpeza química. Uma alternativa para o aumento do fluxo permeado principalmente com soluções de corante, seria uma mudança do método de filtração, para filtrações horizontais, em módulos capazes 
de promover ao efluente altas velocidades tangencias sobre a superfície da membrana, evitando assim o fouling, Degrémont (2007) e Oliveira (2010).

\section{CONCLUSÃO}

Com a realização deste trabalho, foi possível verificar que o processo de separação por membranas pode ser aplicado na remoção da coloração de soluções de corante empregados no tingimento de pele de peixe. Entre as composições estudadas, observou-se-se que houve uma maior remoção de cor da solução de corante preto direto, do que o amarelo reativo. As membranas M2 e M4 foram as que mais se destacaram, com remoções de 47 e $90 \%$, para as soluções de corantes amarelo reativo e preto direto, respectivamente. A membrana M5 foi a que apresentou o maior valor de fluxo permeado com solução de corante $\left(156 \mathrm{~kg} \mathrm{~h}^{-1} \mathrm{~m}^{-2}\right)$ e um baixo índice de fouling. Todas as membranas foram classificadas como sendo de ultrafiltração.

\section{REFERENCIAS}

BASSETI, F. DE. J. Preparação, Caracterização e Aplicação de Membranas Poliméricas Microporosas Assimétricas. 2002. Tese (Doutorado), Universidade Estadual de Campinas, Campinas-SP.

BEZERRA, E. B.; LEITE, A. M. D.; ARAÚJO, E. M.; MÉLO, T. J. A. Obtenção e caracterização de membranas obtidas a partir de blendas poliméricas de poliamida 6. Polímeros: Ciência e Tecnologia, v. 24, n. 3, p. 381-387, 2014.

CHAGURI, M. P.; VIDOTTI; R. M. Utilização de escamas e Eichhornia crassipes no tratamento de efluente de curtume de peles de tilápias. Jaboticabal, 2010.

DEGRÉMONT. Water treatment handbook. $7^{\mathrm{a}}$ edição, Ed. Cedex: Degrémont 2007. Vol. 2.

HABERT, S. C., BORGES, C. P., NOBREGA, R. Processos de Separação por Membranas. COPPE/UFRJ, E-papers, Rio de Janeiro, 2006.

LOWRY, O. H., ROSENBROUGH, N. J., FARR, L.; RANDALL, R. J. Protein Measure with the Folin Phenol Reagent. Journal. Biological Chemistry. v. 193, p. 265-275, 1951.

OLIVEIRA, T. F. de. Tratamento de água de abastecimento público por sistema de separação por membrana de ultrafiltração: estudo de caso na ETA Alto da Boa Vista. São Paulo - SP, 2010.

OURADI, A.; NGUYEN, Q.T.; BENABOURA, A. Polysulfone-AN69 blend membranes and its surface modification by polyelectrolyte-layer deposit-Preparation and characterization. Journal of Membrane Science 454 (2014) 20-35.

PALOMINO-ROMERO, Joel A., LEITE, Otávio M., EGUILUZ, Katlin I. Barrios, SALAZAR-BANDA, Giancarlo R., SILVA, Daniel P, CAVALCANTI, Eliane B.. Tratamento dos efluentes gerados na produção de biodiesel. Ed. Química Nova, 2011.

STREIT, K. F.; FERREIRA, J. Z. Estudo da aplicação de processos de separação com membranas no tratamento de efluentes de curtume: nanofiltração e eletrodiálise. Porto alegre - RS, 2011. 